\title{
Nuevos antecedentes acerca de la presencia de Bombus dahlbomii Guérin-Méneville (Hymenoptera: Apidae) en la Isla Grande de Tierra del Fuego
}

\author{
New antecedents of the presence of Bombus dahlbomii Guérin-Méneville \\ (Hymenoptera: Apidae) in the Big Island of Tierra del Fuego
}

José Díaz Tavie ${ }^{1}$, Lorena Vieli ${ }^{1,2}$ \&

José Montalva ${ }^{1,3}$

El género Bombus comprende alrededor de 250 especies distribuidas en casi todo el mundo (Michener, 2007; Williams et al. 2008). Para Chile se han descrito cuatro especies de abejorros, dos especies recientemente introducidas Bombus ruderatus (Fabricius, 1775) y Bombus terrestris (Linnaeus, 1758) y dos especies nativas Bombus funebris (Smith, 1854) y Bombus dahlbomii (Guerin-Meneville, 1835) (Montalva et al. 2011). En el caso de B. dahlbomii las poblaciones de esta especie han decrecido drásticamente en lugares donde se observó la invasión de abejorros exóticos a partir de los años '90 (Morales et al. 2013; Schmid-Hempel et al. 2013).

B. dahlbomii es la única especie nativa de abejorro de la zona mediterránea y austral de Chile y es la especie del género más grande y meridional del mundo (Cockerell, 1923; Franklin, 1913; Montalva et al. 2011). En base a la bibliografía existente se ha dado como límites de distribución de la especie la región de Coquimbo por el norte y la región de Magallanes por el sur (Franklin, 1913; Montalva et al. 2011, Pérez \& Petersen, 1989; Pérez 2014). Si bien esto, no siempre ha estado del todo claro, por ejemplo Vachal (1906) dio como límite sur la latitud $51^{\circ}$ (Seno de Última Esperanza) y lo mismo Ruiz en su trabajo "El Género Bombus" (1939). Philippi (1862) dió como límite sur la latitud $53^{\circ}$ (Punta Arenas). En 1890 durante la expedición del Buque Albatross a la zona del estrecho de Magallanes, comandada por la comisión de pesca de USA, se reportó la presencia de $B$. dahlbomii en la zona de la Bahía de Borja (Howard, 1890).
Franklin (1913) menciona que la especie es abundante en la zona del estrecho de Magallanes y plantea la posibilidad de que el abejorro este presente en Tierra del Fuego. También cabe señalar que Toro (1986) en la lista preliminar de los ápidos chilenos, da como límite sur para la especie, la región de Carlos Ibáñez del Campo, lo que probablemente fue un lapsus cálami de dicho especialista.

En 1907 Crawshay en su libro Aves de Tierra del Fuego, reporta la existencia de $B$. dahlbomii en la isla grande de Tierra del Fuego, específicamente en el sector Nose Peak Forest [19 Enero 1905] de lo cual hace referencia Cockerell (1923) y posteriormente Michener (2007). El ejemplar se encuentra depositado en el Museo Británico de Londres (Cockerell, 1923).

Además cabe mencionar que el ejemplar más austral capturado en la región de Magallanes, se encuentra depositado en el American Museum of Natural History y corresponde a una reina de B. dahlbomii colectada en la Isla de Navarino [AMNH_BEE00147245; Reina; Isla Navarino, Chile; Septiembre 1935; J. Bird].

El fin de esta nota es reportar y revalidar la presencia de $B$. dahlbomii en la isla grande de Tierra del Fuego, debido a la poca representatividad

\footnotetext{
1 Salvemos Nuestro Abejorro.

2 Instituto de Medio Ambiente, Universidad de la Frontera, Temuco, Chile.

montalva.Jose@yahoo.es $>$
} 
en museos y la vaga información de esta especie para esta zona en particular.

En base a ejemplares colectados, material fotográfico y observaciones en terreno, se registraron 29 reinas y 2 obreras de $B$. dahlbomii, en las zonas de Porvenir, Primera Angostura y el tramo comprendido entre Puerto Arturo y río Woodsend (Fig. 1). Los datos de geolocalización de los ejemplares fotografiados se tomaron con GPS Garmin Etrex Vista HCX (datum WGS 84).

Registros:

1.- $54^{\circ} 02$ ' 31" S $70^{\circ} 03^{\prime} 15^{\prime \prime} \mathrm{W}, 2 \mathrm{~km}$ al de Puerto Arturo, 02 Nov 2014. (4 reinas, fotografiadas, Fig. 2)

2.- $53^{\circ} 58^{\prime} 06^{\prime \prime} \mathrm{S} 70^{\circ} 05^{\prime} 33^{\prime \prime} \mathrm{W}$, Rio Condor 03 Nov 2014. (6 reinas, fotografiadas)

3.- $53^{\circ} 51^{\prime} 29^{\prime \prime} \mathrm{S} 70^{\circ} 09^{\prime} 28^{\prime \prime} \mathrm{W}$, Puerto Yartou, 06 Nov 2014. (3 reinas, fotografiadas)

4.- $53^{\circ} 52^{\prime} 13^{\prime \prime} \mathrm{S} 70^{\circ} 09^{\prime} 07$ " W, Rio Caleta, 07 Nov 2014. (4 reinas, fotografiadas)

5.- $53^{\circ} 46^{\prime} 24^{\prime \prime}$ S $70^{\circ} 09^{\prime} 36^{\prime \prime} \mathrm{W}$, Puesto
Maria, 08-09 Nov 2014. (7 reinas, fotografiadas, Fig. 2)

$$
\text { 6.- } 53^{\circ} 46^{\prime} 14^{\prime \prime} \text { S } 70^{\circ} 09^{\prime} 16^{\prime \prime} \mathrm{W} \text {, Caleta }
$$

Maria, 11 Nov 2014. (4 reinas, fotografiadas)

7.- $52^{\circ} 29^{\prime} 39^{\prime \prime} \mathrm{S} 69^{\circ} 26^{\prime} 48^{\prime \prime} \mathrm{W}, 5 \mathrm{~km} \mathrm{E}$ del cruce de Primera Angostura, 18 Ene 2008 (2 obreras, colectado por Eduardo Faúndez, Colección personal)

8.- $53^{\circ} 17^{\prime} 47^{\prime \prime} \mathrm{S} 70^{\circ} 22^{\prime}$ 5" W, Porvenir, Sep 2008 (1 reina, colectado por Eduardo Faúndez, Colección personal)

Todos los ejemplares fotografiados se observaron en sectores resguardados del viento imperante de la zona, asociados a cuerpos de agua dulce y con un mayor grado de vegetación, las reinas se pudieron observar forrajeando en Berberis microphylla Forst, Ribes magellanicum Poir, Embothrium coccineum J.R.Forst. \& G.Forst., Berberis ilicifolia Forst, Taraxacum officinale F.H. Wigg y Fuchsia magellanica Lam, lo que concuerda con las asociaciones florales de la parte continental Magallánica (Pérez, 2014).

Los reqistros de esta nota coinciden con

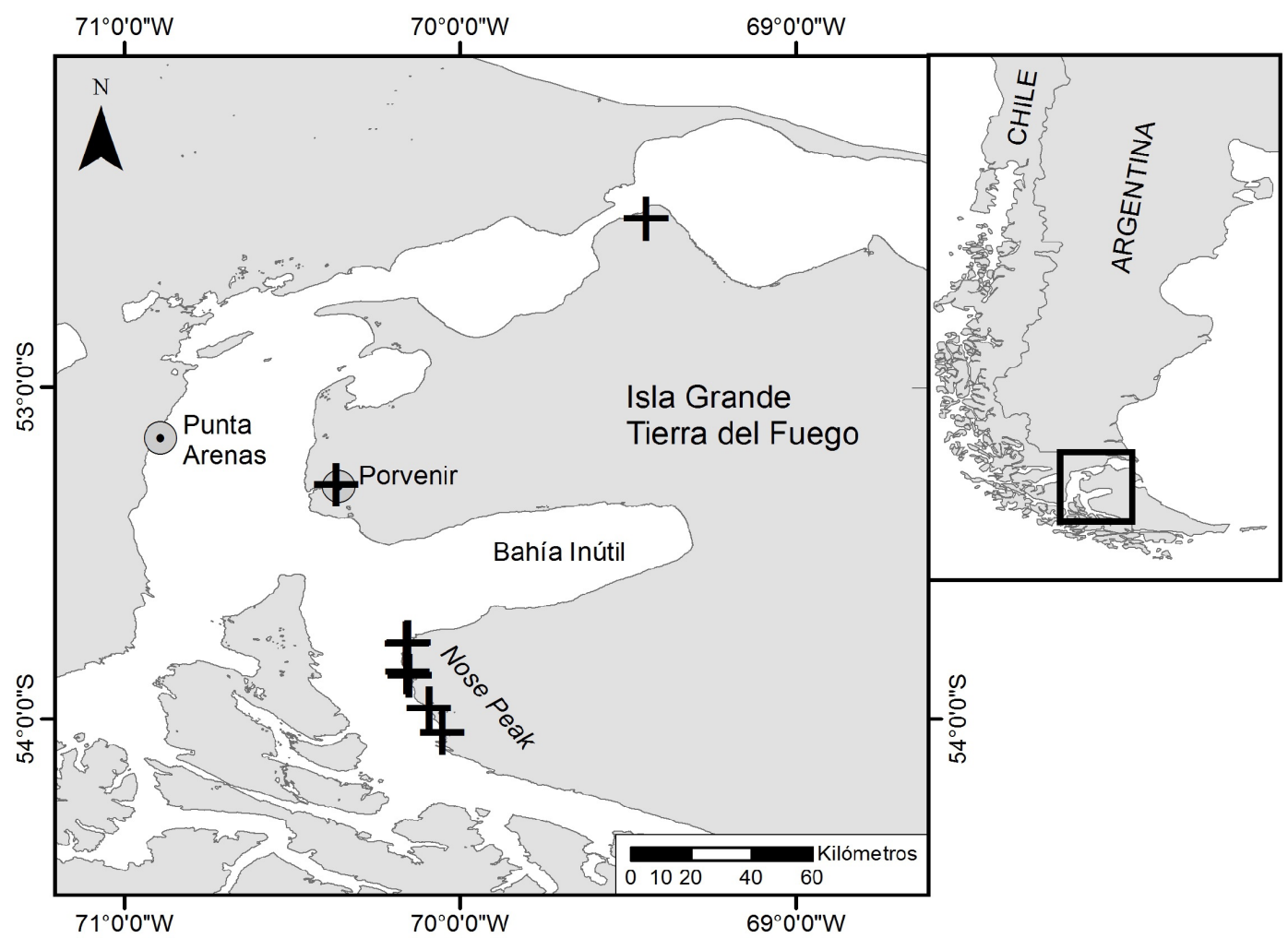

Fig. 1. Localización (cruz) de registros de Bombus dahlbomii en Tierra del Fuego. 

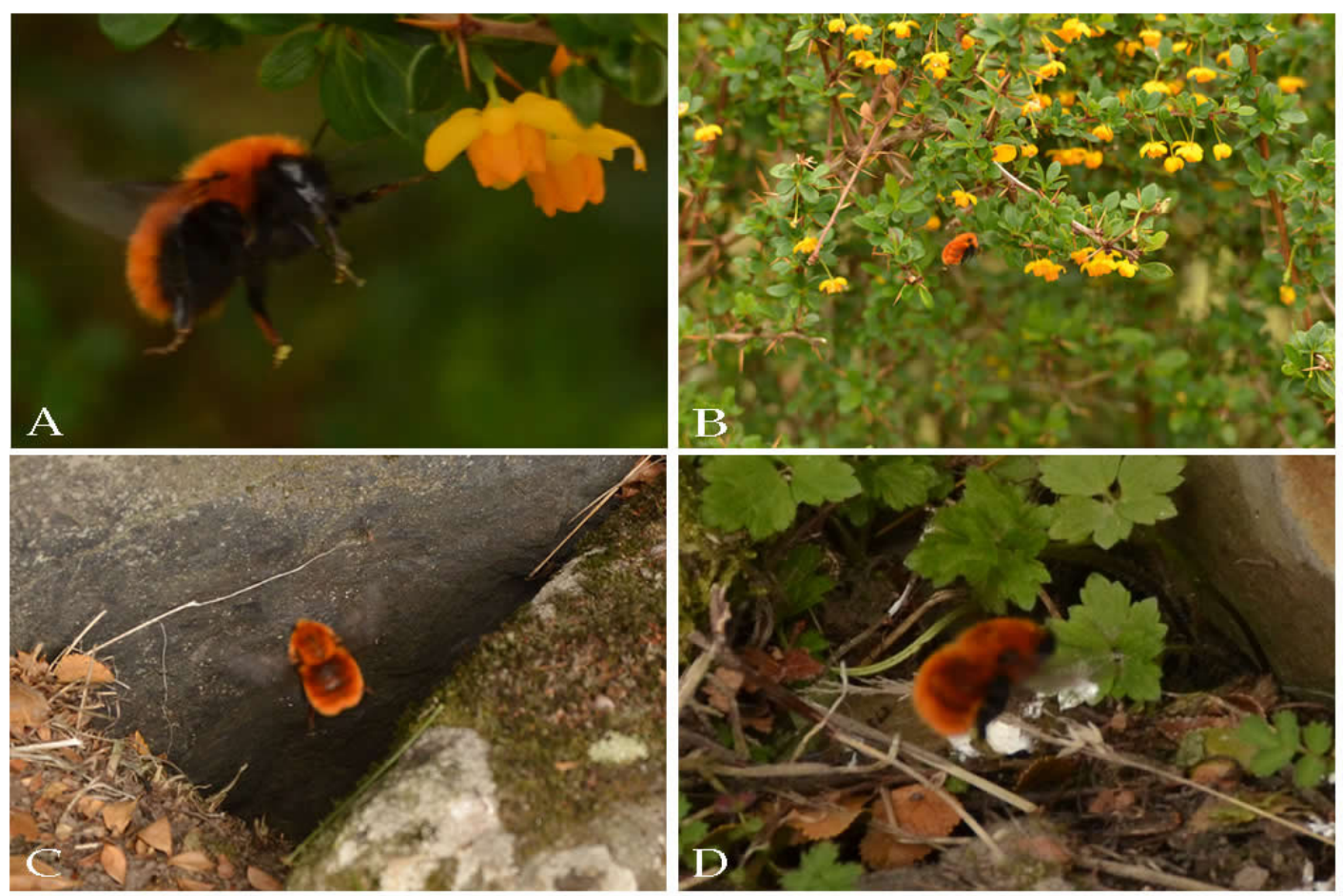

Fig. 2. Bombus dahlbomii A, B forrajeando sobre calafate Berberis microphylla en el sector de Puerto Arturo C, D a la entrada del nido sector de Puerto María.

la ubicación de los individuos mencionados por Crawshay en Nose Peak Forest 1905, además se reportan como nuevas localidades Primera Angostura y Porvenir (Fig. 1), lo que permiten confirmar la presencia de esta especie en Tierra del Fuego. Más reportes y exploraciones a futuro son necesarias para establecer la real distribución de B. dahlbomii, tanto en la isla grande de Tierra del Fuego, como también en sectores aledaños como el Parque Nacional Alberto de Agostini, Isla Dawson, Isla Navarino y el Parque Nacional Cabo de Hornos.

\section{AGRADECIMIENTOS}

Agradecemos a Eduardo Faúndez y Vicente Pérez por proporcionarnos valiosa información.

\section{LITERATURA CITADA}

Crawshay, R. (1907). The birds of Tierra del Fuego. London: B. Quaritch.

Cockerell, T.D.A. (1923). Descriptions and records of bees XCVIII The Annals and Magazine of Natural History, 9(12), 238-247

Franklin, H.J. (1913). The Bombidae of the New World. Transactions of the American Entomological Society, 39, 73-200

Howard, L.O. (1890). Annotated catalogue of the insects collected in 1887-'88. Proceedings of the United States National Museum. $12,185-216$.

Michener, C.D. (2007). The bees of the world. Second edition. John Hopkins University Press, Baltimore.

Montalva, J., Dudley, L., Arroyo, M.K., Retamales, H. \& Abramovich, A.H. (2011). Geographic distribution and associated flora of native and introduced bumble bees (Bombus spp.) in Chile. Journal of Apicultural Research, 50(1), 11-21.

Morales, C.L., Arbetman, M.P., Cameron, S.A. \& Aizen, M.A. (2013). Rapid ecological replacement of a native bumble bee by invasive species. Frontiers in Ecology and the Environment, 11(10), 529-534.

Pérez, V. \& Petersen, J. (1989). Notas sobre abejas de la Región Magallánica, 
Chile (Hymenoptera: Apoidea). Acta Entomológica Chilena, 15, 257-260.

Pérez, V. (2014).Contribución a la compilación de registros de plantas asociadas a Bombus (Fervidobombus) dahlbomii Guérin Méneville (Hymenoptera: Apidae) en Chile. Anales Instituto Patagonia, 42(1), 81-85.

Philippi, R.A. (1862). Sobre algunos insectos de Magallanes. Anales de la Universidad de Chile, 21, 407-416.

Ruiz, F. (1940). El género Bombus Latr. Revista Chilena de Historia Natural, 43, 106-110.

Schmid-Hempel, R., Eckhardt, M., Goulson, D., Heinzmann, D., Lange, C., Plischuk, S., Ruz L., Scriven, J. J. \& Schmid-Hempel, P. (2013). The invasion of southern South America by imported bumblebees and associated parasites. Journal of Animal Ecology, 83, 823-837.

Toro, H. (1986). Lista preliminar de los ápidos chilenos (Hymenoptera: Apoidea). Acta Entomológica Chilena, 13, 121-132.

Vachal, J. (1906). Zoologie: Insectes. Hyménoptères. Apidae. Résultats du Voyage du S.Y. Belgica en 1897-18981899 sous le commandement de A. de Gerlache de Gomery: Rapports Scientifiques (1901-1913). Buschmann: Anvers. 63-64 pp.

Williams, P.H., Cameron, S.A., Hines, H.M., Cederberg, B. \& Rasmont, P. (2008). A simplified subgeneric classification of the bumblebees (genus Bombus). Apidologie, 39, 46-74. 